Check for updates

Cite this: Mater. Adv., 2021, 2, 6676

Received 28th July 2021,

Accepted 12th September 2021

DOI: 10.1039/d1ma00656h

rsc.li/materials-advances

\title{
Fabrication of an autonomously self-healing flexible thin-film capacitor by slot-die coating $\dagger$
}

\author{
Susanna Vu, $\ddagger^{\mathrm{a}}$ Gnanesh Nagesh, $\ddagger^{\mathrm{b}}$ Nastaran Yousefi, $\ddagger^{\mathrm{a}}$ John F. Trant, (D) ${ }^{\mathrm{a}}$ \\ David S. - K. Ting, (D) ${ }^{\mathrm{b}}$ M. Jalal Ahamed (D) *b and Simon Rondeau-Gagné (D) *a
}

\begin{abstract}
Flexible pressure sensors with self-healing abilities for wearable electronics are being developed, but generally either lack autonomous self-healing properties or require sophisticated material processing methods. To address this challenge, we developed flexible, low-cost and autonomously self-healing capacitive sensors using a crosslinked poly(dimethylsiloxane) through metal-ligand interactions processed into thin films via slot-die coating. These films have excellent self-healing properties, approximately $1.34 \times 10^{5} \mu \mathrm{m}^{3}$ per hour at room temperature and $2.87 \times 10^{5} \mu \mathrm{m}^{3}$ per hour at body temperature $\left(37^{\circ} \mathrm{C}\right)$. Similarly, no significant change in capacitance under bending strain was observed on these flexible thin-films when assembled on poly(ethyleneterephthalate) (PET) substrates; capacitors showed good sensitivity at low pressure regimes. More importantly, the devices fully recovered their sensitivity after being damaged and healed, which is directly attributed to the rapid and autonomous self-healing of the dielectric materials.
\end{abstract}

\section{Introduction}

Through the Internet of Things (IoT), numerous household, consumer, and industrial objects are constantly acquiring and transmitting a large amount of data wirelessly, enabling new communication modes between humans and our possessions. ${ }^{1,2}$ Through this avenue of data collection and transmission, the IoT is inventing ever-emerging opportunities for more direct integration of the physical world via the introduction of various sensors into computer-based systems in many industry-oriented and user-specific applications. ${ }^{3,4}$ It is expected that the IoT will deliver significant economic benefits, enable technological improvements, and reduce human exertion. ${ }^{5}$ Together, this will improve human productivity by automating evermore tedious and mundane tasks. Important progress has been made in multiple scientific areas toward next generation electronics capable of being molded in different shapes and forms, and that can be utilized directly on (or inside) the human body to enhance our connectivity to the environment. Ultimately, new applications such as personalized healthcare and large-scale energy production can be

\footnotetext{
${ }^{a}$ Department of Chemistry and Biochemistry, University of Windsor, Ontario, N9B 3P4, Canada. E-mail: srondeau@uwindsor.ca

${ }^{b}$ Department of Mechanical, Automotive and Materials Engineering, University of Windsor, Windsor, Ontario, N9B 3P4, Canada. E-mail: M.Ahamed@uwindsor.ca $\dagger$ Electronic supplementary information (ESI) available: Detailed experimental procedures for all new compounds, and a complete characterization of materials and devices. See DOI: $10.1039 / \mathrm{d} 1 \mathrm{ma} 00656 \mathrm{~h}$

\$ These authors contributed equally to this work.
}

developed through this progress. ${ }^{6-10}$ Among others, capacitive devices and sensors are becoming an integral part of nextgeneration electronics and are particularly promising for the detection of multiple stimuli, including pressure. ${ }^{11-13}$ In recent years, a plethora of stretchable/flexible capacitors have been fabricated with various materials. ${ }^{14-16}$ A capacitor is formed when two parallel plate electrodes are separated by a dielectric. The change in the separation gap (dielectric layer) between the top and bottom electrode, as well as the dielectric material nature can dramatically enhance the capacitive sensing. This constitutes a particularly promising platform to develop new materials and processing techniques to trigger innovative properties in electronics.

Among these new properties, autonomous self-healing attracts a lot of attention for the fabrication of next-generation electronics. ${ }^{17,18}$ Defined as the capability of a material to regenerate and restore partially or completely its initial properties after suffering from damages (strain, puncture, cracks, etc.), self-healing can be intrinsic to a given material, or require external stimuli (such as heating or pressure) to be triggered. ${ }^{19}$ Particularly promising for the development of advanced electronics and biotechnology, there has been a constant effort to develop novel strategies and materials designs to enable rapid and autonomous self-healing, without the use of external triggers or complex methodology. ${ }^{20-23}$

The literature on soft pressure sensors from capacitors is rich, with many reports of high-performance devices prepared from a multitude of materials that have been comprehensively reviewed throughout recent years. ${ }^{24,25}$ Additionally, high-performance 
pressure sensors from other types of devices, including organic field-effect transistors, and high-performance dielectric materials have also been reported. ${ }^{26,27}$ However, the incorporation of selfhealing materials into electronic devices is much less common despite being particularly attractive to improve their operational lifetime, especially in applications where they are susceptible to wear and fractures due to different movements. In recent years, several researchers reported the fabrication of flexible sensors with self-healing materials/properties. Among others, Bao and co-workers reported an elastic autonomous self-healing capacitive sensor based on a combination of dynamic metal-coordinated bonds and hydrogen bonds as a dielectric layer that could achieve up to $98 \%$ of its healing efficiency after 48 hours at $25{ }^{\circ} \mathrm{C}$ and demonstrated good electrical recovery. ${ }^{28}$ In another study, Wang et al. developed a pressure sensor using a ternary composite polymer system with high stretchability, self-healing properties, and solution-processing compatibility that demonstrated a pressure sensitivity of $37.6 \mathrm{kPa}^{-1}$ for low pressure range of $0-0.8 \mathrm{kPa} .{ }^{29}$ Other examples of self-healing pressure sensors have been reported using hydrogels. For example, Zhu et al. used a smart ionic gelatin/polyacrylamide/nano-clay hydrogel with high conductivity, robust adhesion properties and self-healing properties to design self-healing capacitive sensors. ${ }^{30}$ The resulting sensors showed good sensitivity $\left(0.11 \mathrm{kPa}^{-1}\right)$, and the capacitance responded up to $2 \mathrm{kPa}$ in a largely linear manner. More importantly, the devices prepared from the hydrogel materials showed rapid self-healing, reaching a self-healing efficiency of $85 \%$ after $60 \mathrm{~min}$. Despite the emergence of numerous new materials based on a myriad of self-healing strategies (encapsulation of healing agents, dynamic crosslinking, shape-memory effects), self-healing electronics remain a relatively emerging field; new materials and fabrication processes need to be fully integrated toward autonomously self-healing electronic devices capable of being manufactured at low cost.

Recently, our team developed a novel self-healing material based on poly(dimethylsiloxane) (PDMS) (Fig. 1a). ${ }^{31}$ Chemically designed to enable an autonomous intrinsic self-healing at the molecular level, dynamic imine and metal-ligand bonds were incorporated in a commercially available PDMS derivative to generate the new materials. The unique property of this material is that it can be prepared in large-scale at low cost and finetuned in terms of thermomechanical properties by a careful selection of the metal center used in the supramolecular crosslinking. ${ }^{32}$ Capacitive pressure sensors and microfluidic devices were fabricated using this material, and the devices showed particularly efficient self-healing at room temperature (Fig. S2, ESI $\dagger)^{31,33}$ While self-healing devices were demonstrated in previous work with our new materials, the fabrication of thin-film electronics, such as capacitive or resistive devices, remains a challenge, as self-healing is a phenomenon highly dependent on the polymer segmental mobility. ${ }^{34,35}$ Given the need for thin and ultrathin layers in these devices, high selfhealing efficiencies can be difficult to achieve in these electronics due to the small amount of materials being present to restore the damaged area and solid-state properties. Moreover, the integration of the self-healing materials into electronic devices can be challenging due to poor materials compatibility a.

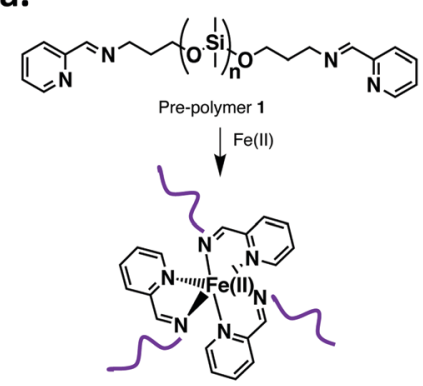

C.
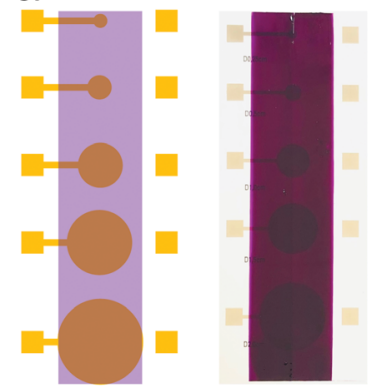

Fig. 1 (a) Crosslinking of pre-polymer 1 with Fe(॥) metal ions; (b) slot-die coater used for printing films on PET substrate; (c) schematic (left) and the actual (right) PET substrate with dielectric film (polymer 1) printed on top, and (d) schematic (left) and the actual (right) PET substrate with dielectric (polymer 1) and top electrode (PEDOT:PSS) films printed on top. Yellow, purple, and blue colors correspond to silver ink, dielectric (polymer 1), and PEDOT:PSS respectively.

and the difficulty of processing on different substrates. Therefore, new materials and methods for the fabrication of autonomous self-healing thin film devices are still highly desirable.

Herein, we report the fabrication of new autonomous selfhealing capacitive sensors based on an intrinsic self-healing dielectric. In order to fabricate the materials in the most straightforward and reliable manner possible, a poly(ethyleneterephthalate) (PET) substrate coated with silver ink was utilized. The dielectric self-healing materials was then deposited as a thin layer on top of this platform via slot-die coating (Fig. 1b), which allowed for a uniform and controlled deposition of the self-healing dielectric. To finalize the fabrication of the device, PEDOT:PSS, a conductive polymer known for its selfhealing capabilities, was printed using slot-die coating. ${ }^{36}$ The resulting capacitors, depicted in Fig. 1c and d, were characterized extensively through multiple techniques in order to fully unveil their electronic properties before and after healing. Moreover, given that self-healing in thin films can be challenging, a detailed characterization of the materials' self-healing was performed through optical microscopy. The resulting devices were found to be completely flexible/bendable without any compromise to their electronic properties after complete damage and self-healing. Additionally, the application of the new thin-film capacitors in pressure sensing was demonstrated. The new self-healing electronics can be manufactured rapidly at low cost, thus opening new avenues for self-healing electronics to be integrated into various platforms for a diverse number of applications. 


\section{Results and discussion}

\subsection{Materials preparation and processing}

To fabricate the self-healing capacitive sensors, a self-healing PDMS-based dielectric material was prepared according to our previously reported procedures. ${ }^{31,32}$ Briefly, a pre-polymer containing dynamic imine bonds was prepared from the condensation of aminopropyl-terminated PDMS and pyridine 2carboxaldehyde. The pre-polymer was then crosslinked by generating intermolecular supramolecular metal-ligand interactions through metal coordination with $\mathrm{Fe}\left(\mathrm{BF}_{4}\right)_{2}$ to afford the self-healing dielectric, polymer 1 (Fig. 1a). Based on previous reports, $\mathrm{Fe}(\mathrm{II})$ was chosen as metal crosslinker given its optimal thermomechanical properties (resistance to fracture and Young's modulus) and good bulk self-healing efficiency. However, it is important to note that this system can be completely fine-tuned as the self-healing efficiency and resistance to fracture can be directly rationally controlled and predicted by coordination bond lengths (strength of the $\mathrm{M}-\mathrm{L}$ interactions) and size of the counter-ions. Upon preparation of the materials, a solution was prepared in chloroform in order to further process the materials in thin films. Slot-die coating was selected to prepare and process the different layers of the printed capacitor as it is particularly advantageous for a rapid patterning of soft substrates with minimal wastes. Fig. $1 \mathrm{~b}$ presents a schematic of the slot die coating instrument used for the dielectric deposition (polymer 1).

To prepare the self-healing device, a PET substrate was coated with silver inks prior to dielectric deposition. Finally, PEDOT:PSS top electrode, a material known for its versatility and self-healing properties, was deposited via slot-die coating on top of the dielectric layers. The detailed procedure for the deposition of different materials through slot die coating and their properties is provided in the ESI. $\dagger$ The images of slot-die coated dielectric polymer 1 with and without the PEDOT:PSS electrode on the PET substrate are displayed in Fig. 1c and $\mathrm{d}$ respectively. The purple film represents the self-healing dielectric (polymer 1), whereas the coating blue film is PEDOT:PSS. As shown in the images, the deposited materials have a consistent coverage over the area of interest.

\subsection{Thin film self-healing}

Self-healing mechanisms are often based on the generation of mobility; the damaged area (crack, hole or scratch) can be healed only once a mobile polymer phase is generated that can close the crack or scratch. ${ }^{34}$ Mostly evaluated in bulk, selfhealing in a thin film can therefore be more challenging as the quantity of materials is limited and the generation of mobile polymer chains can be more difficult. Therefore, to gain insight on the self-healing behavior of the dielectric materials in thin films, the self-healing property of polymer 1 was first investigated directly on the PET platform. In order to generate damage, the layered assembly was immersed in liquid nitrogen $\left(-78{ }^{\circ} \mathrm{C}\right)$ for a few seconds. Using this approach, we were able to introduce cracks with different depths and widths (average depth and width of $435.2 \mu \mathrm{m}$ and $20.5 \mu \mathrm{m}$ respectively in a.

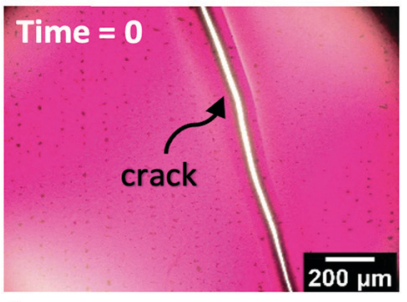

c.

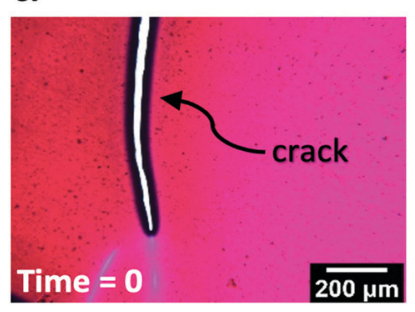

b.

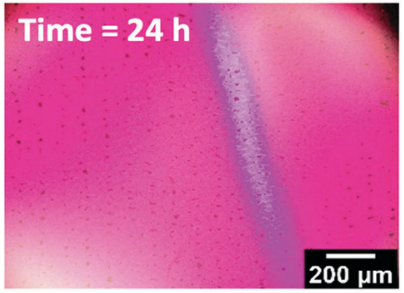

d.

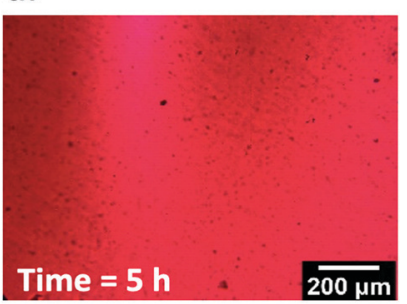

Fig. 2 Self-healing of polymer 1 in thin films visualized by optical microscopy (a) before and (b) after 24 hours of healing at room temperature, and (c) before and (d) after 5 hours of healing at human body temperature $\left(37^{\circ} \mathrm{C}\right)$.

$0.09 \mathrm{~cm}^{2}$ ) into the coated film. Following the generation of structural damage, the cracked films were imaged using an optical microscope and the healing process was monitored over 24 hours to establish the efficiency of material self-healing at room temperature, and the results are depicted in Fig. 2a and b. Within only 10 hours, the damaged area (crack) had already substantially closed (meaningful gap coverage) (see ESI, $\dagger$ Fig. S3 and Video S1) and was fully healed after 24 hours at room temperature. The self-healing efficiency of dielectric films was further studied at human body temperature, $37{ }^{\circ} \mathrm{C}$, to probe its potential in wearable electronics. Fig. $2 \mathrm{c}$ and $\mathrm{d}$ shows the optical microscope images of the cracked dielectric film and the healed film. As shown in Fig. 2d, there is a significant increase in self-healing efficiency of dielectric material at $37{ }^{\circ} \mathrm{C}$ and the film is healed completely after only 5 hours. Additionally, the dielectric film self-healing efficiency was studied at $50{ }^{\circ} \mathrm{C}$ and showed significant gap coverage over the course of 2 and 7 hours for the gap width of approximately $12 \mu \mathrm{m}$ and $32 \mu \mathrm{m}$ with $435.2 \mu \mathrm{m}$ depth (Fig. S5, ESI $\dagger$ ). This improvement of the self-healing kinetic is not unexpected, as an increase temperature will promote the generation and flow rate of the mobile polymer phase, thus favoring the restoration of the initial morphology. The optical microscope images of the crack at different time intervals prior to full healing is provided in ESI, $\dagger$ Fig. S3-S5. Additionally, atomic force microscopy was used to probe for the nanoscale morphology, which confirmed that the films are smooth and do not show any metal clusters or aggregated domains (Fig. S6, ESI $\dagger$ ). Overall, this evaluation of the self-healing kinetics at room and human body temperature demonstrates the efficiency of the self-healing in thin films and confirms the strong potential of polymer $\mathbf{1}$ for wearable electronic applications.

Upon self-healing characterization of the dielectric film on the PET platform, a PEDOT:PSS electrode of was deposited 
(thickness of $74.6 \pm 7 \mathrm{~nm}$ measured by profilometer) on top of the dielectric through slot-die coating. Importantly, PEDOT:PSS was chosen as a soft electrode to complete the capacitor's structure given its good conductivity, favorable thermomechanical properties and previously reported self-healing properties. In order to achieve a smooth and uniform film, an aqueous suspension of PEDOT:PSS was mixed with polyethylene glycol and glycerol in 2-propyl alcohol, and was further printed on the layered assembly. ${ }^{37}$ The complete procedure for depositing the PEDOT:PSS layer is detailed in ESI. $\dagger$

\subsection{Device characterization}

Upon characterization of the printed layer assembly and confirmation of successful self-healing of the dielectric layer at the microscale, the new fully printed thin-film capacitors were characterized to investigate how the capacitance values change in response to pressure. First, we investigated the impact of bending on device performance. The results are summarized in Fig. 3. Devices were put under bending strain and their capacitance was measured. As shown in Fig. 3a, the capacitance measurements were carried out for devices with different surface areas under three different points along the stress-release process temporal axis: before applying any bending strain in the flat position, under bending strain and in the curved position, and after releasing the bending strain and back in the flat position. When the capacitor is curved (concave) and is under strain, the top electrode is expected to experience higher stress (stretching) than the dielectric layer and the bottom electrode. This increased force on the top electrode can potentially lead to crack formation and propagation upon bending strains, resulting in a general decrease in device performance. Notably, for our new self-healing capacitors, no significant change in capacitance was observed under bending strain. This was true regardless of the area of the capacitors. Capacitors with surface area of $0.25,0.5$ and $1.0 \mathrm{~cm}^{2}$ show an average capacitance value between 3.0 and $3.5 \mathrm{pF}$, which is typical for capacitors made from similar elastomeric materials. ${ }^{16}$ For the capacitors with electrode area of $1.5 \mathrm{~cm}^{2}$, a slightly higher value of capacitance was measured (4.5 pF). More interestingly, in cases where the capacitance increases $\left(1.0 \mathrm{~cm}^{2}\right)$ or decreases $\left(1.5 \mathrm{~cm}^{2}\right)$ upon bending, the values are restored immediately after the release of the bending strain. This tolerance to strain can be attributed to the loading and unloading linearity of the elastic modulus showing favorable mechanical properties of PEDOT:PSS and to the high deformability of the different layers. Nonetheless, all printed capacitors showed good tolerance to mechanical stress and stable capacitance values, independent of the bending mode.

After evaluating the effect of bending strain on the device performance, devices were subjected to pressure tests. To achieve this, a pressure, ranging from 0 to $2.0 \mathrm{kPa}$, was applied by loading a series of weights ( 1 to 20 grams) on capacitors and monitoring the change in the capacitance values. Pressure is defined as force divided by the area, so the applied pressures were calculated based on the mass and surface area of the weights used. The capacitance variation upon applied pressure has been evaluated for different capacitor areas, and the results are depicted in Fig. $3 \mathrm{~b}$ and Fig. S7 (ESI $\dagger$ ). In all the studied cases, the capacitance change is more significant in the lowpressure range $(0-1 \mathrm{kPa})$ and becomes less sensitive at higher pressure ranges $(1-2 \mathrm{kPa})$. This can be attributed to the low thickness of the dielectric layer which makes it less sensitive to deformations at high pressures, exhibiting a clear ceiling effect. To obtain a better comparison with other capacitive pressure sensors, the pressure sensitivity of the self-healing capacitors was calculated. The pressure sensitivity $(S)$ can be defined as the slope of the traces in Fig. $3 \mathrm{~b}\left(S=\Delta\left(C-C_{0}\right) / \Delta p\right)$, where $p$ denotes to the applied pressure, and $C$ and $C_{0}$ are the capacitances with and without applied pressure, respectively. The pressure sensitivity of the sensors with 0.25 and $1.5 \mathrm{~cm}^{2}$ areas are 0.15 and $0.23 \mathrm{kPa}^{-1}$ for the $0-1 \mathrm{kPa}$ range and 0.02 and $0.05 \mathrm{kPa}^{-1}$ for $1-2 \mathrm{kPa}$ range, respectively. As the sensor area increases from 1.5 to $2.0 \mathrm{~cm}^{2}$, the pressure sensitivity significantly decreases to $0.09 \mathrm{kPa}^{-1}$ for the $0-1 \mathrm{kPa}$ and $0.03 \mathrm{kPa}^{-1}$ for $1-2 \mathrm{kPa}$ range (Fig. $3 \mathrm{~b}$ and Fig. S6, ESI $\dagger$ ). Although the highest sensitivity, $2.9 \mathrm{kPa}^{-1}$, for the lowpressure range $(0-1 \mathrm{kPa})$ belonged to the sensor with $1.0 \mathrm{~cm}^{2}$ area (Fig. S6, ESI $\dagger$ ); this sensor had the lowest sensitivity, $0.02 \mathrm{kPa}^{-1}$, at the higher-pressure range (1-2 kPa). These results demonstrate that an increase in the capacitor area leads to a decrease in sensor sensitivity. Importantly, this decrease is more prominent in the lower pressure domain as illustrated by an almost two orders of magnitude drop in sensitivity when the sensor area goes from $1.0 \mathrm{~cm}^{2}$ to $2.0 \mathrm{~cm}^{2}(0-1 \mathrm{kPa}$ regime). As a.

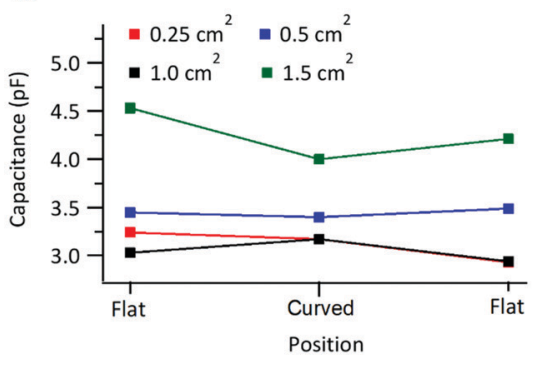

b.

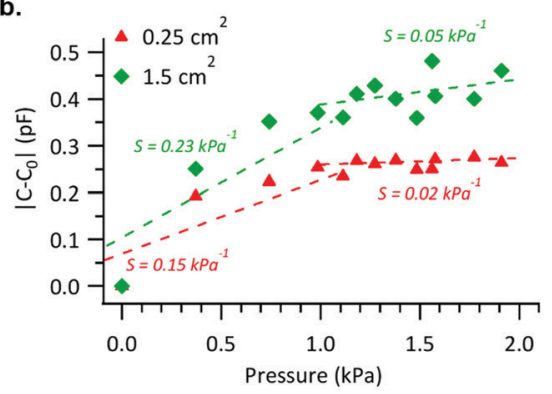

Fig. 3 (a) Static measurement of capacitance as a function of curvature for flat $(1 / r \approx 0)$ and curved $(1 / r=0.17)$ positions for different capacitor areas, and (b) representative capacitance variation as a function of applied pressure, for capacitors with 0.25 (red) and $1.5 \mathrm{~cm}^{2}$ (green) electrode area. 
expected, an increase in capacitive sensor area increases the capacitance response, whereas a decrease in aspect ratio (width to length ratio) reduces the linearity response of the sensor. ${ }^{38,39}$ Importantly, this analysis confirms that the form factors of the capacitor can be carefully fine-tuned to either increase the sensor sensitivity or its linearity. Additionally, the variation in the capacitance upon different applied pressure is an indication that the PEDOT:PSS top electrode is fully compressed and, as a result, pressure is sensed by the dielectric layer. Consequently, the change in the dielectric thickness (reduced thickness) results in the change in the capacitance. This further clarifies the decrease in sensor sensitivity in the lower pressure range $(0-1 \mathrm{kPa})$ as the capacitive sensor area increases.

In addition to this in-depth characterization of the sensors and sensitivity towards different pressures, dynamic pressure measurements were performed to confirm the devices' ability to respond in real time. This is particularly important for the development of novel stretchable and conformable electronics. The measurement was performed by successively putting pressure on the printed sensors with the fingertip over 20 seconds. As shown in Fig. S8 (ESI $\dagger$ ), the printed capacitive sensors show fast and reproducible response to various pressure, which confirms the sensors capability to detect dynamic pressure changes.

\subsection{Self-healing capacitors}

After characterizing the sensitivity of the new printed capacitors towards mechanical deformation and pressure, a detailed investigation of the self-healing of the sensor was performed. This was examined by damaging the device and monitoring the change in the capacitance values as a function of time. As shown in Fig. 4a, the printed thin-film device was shown to undergo rapid self-healing, reaching its original capacitance values after only 250 minutes (4 hours) at room temperature. Although the healing process appears to be slower in the beginning, based on the measured capacitance values, it dramatically increases after 200 minutes (approximately 3 hours). This behavior can be explained by the generation of the mobile phase; generation of the mobile polymer phase involves an initial induction period before a sufficient amount of polymer migrates to the damage site to create a thick-enough dielectric layer to recover capacitance. Once the connection is reformed, capacitance is rapidly restored.

Following the characterization of the self-healing properties, we also examined the impact of curvature on the capacitor performance and the restoration of its capacitance values after bending. Based on the static measurements shown in Fig. 4b, the convex bending of the capacitor does not impact the magnitude of the capacitance significantly; in the case of the pristine films, the capacitance increases only $11 \%$ when it is in the bend position and is nearly fully restored after returning to its original flat position. The minor increase in the capacitance at the convex position can be explained by the relationship of the capacitance with the dielectric thickness $\left(\propto \frac{1}{d}\right)$ and surface area $(\propto A)$ in a parallel-plate capacitor. When bended in a convex position, the dielectric layer is contracted to some degrees, resulting in a thickness increase (decrease in capacitance). Additionally, the same tests were carried out for devices with healed dielectric films to confirm that the device performance recovers after healing. As demonstrated in Fig. $4 \mathrm{~b}$, the convex bending of the device with healed dielectric film decreases the capacitance by $17 \%$ and the capacitance is not restored after returning to its original flat position. This result, contrasting to those from non-damaged devices, can be attributed to a non-complete healing of the device, potentially causing some of the cracks to open up again and prolong their healing process. The increase in capacitance after crack formation was also observed previously and is shown in Fig. 4a. Conversely, the capacitance is impacted notably in concave position and decreases almost $30 \%$ from its original values for the device with pristine films. Moreover, the capacitance does not restore to its initial values once returned to the flat position and even decreases further ( $44 \%$ of its original values). The observed steady decrease in capacitance can be explained by an irreversible damage to the device due to concave bending. At the concave position, different layers of the capacitor experience different degrees of stretching. Given the difference in materials deformability, and considering the relative brittleness of the silver electrodes, permanent cracks can be formed within the silver film when bended in the concave position. As a result, the capacitance values decrease even after the capacitor
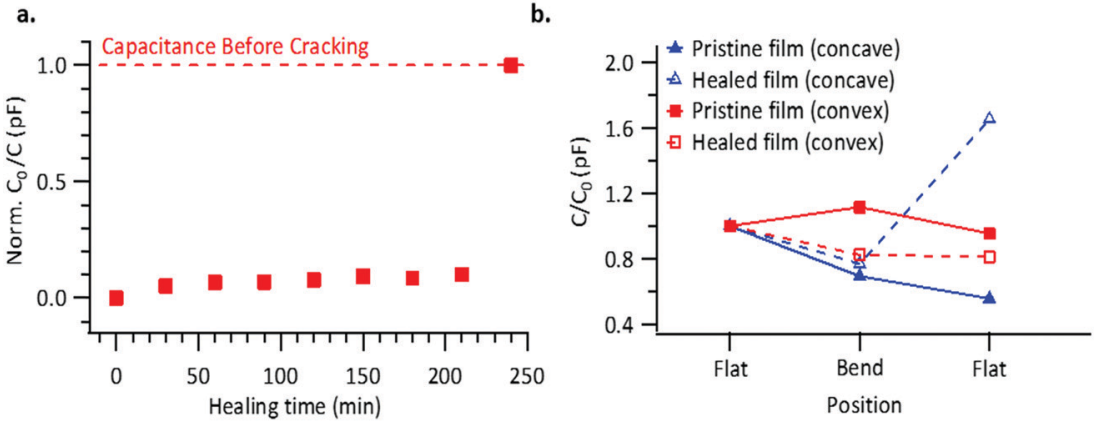

Fig. 4 (a) Evolution of the capacitance during the healing process measured for an area of $1.0 \mathrm{~cm}^{2}$. (b) Static capacitance measurement of pristine and healed films in both concave and convex positions. 
Table 1 Performance comparison of the new printed self-healing pressure sensors with few previously reported devices

\begin{tabular}{|c|c|c|c|c|c|}
\hline Materials & Method of fabrication & Dielectric thickness $(\mu \mathrm{m})$ & Sensitivity $\left(\mathrm{kPa}^{-1}\right)$ & Healability & Ref. \\
\hline Thermoplastic polyurethane & 3D printing & 200 & 50 & NA & 45 \\
\hline Graphene oxide & Spray-coating & 300 & 0.8 & NA & 46 \\
\hline PDMS & Spin-coating & 400 & 1000 & NA & 16 \\
\hline Imine-based PDMS (this work) & Slot-die coating & 85 & 2.9 & Autonomous & - \\
\hline
\end{tabular}

is returned to the flat position. Similarly, the capacitance of the device with the healed dielectric film decreases when it is in the concave position, but to a lesser extent ( $23 \%$ of the initial value) compared to the device with pristine film. Surprisingly, the capacitance increases after returning to the flat position and reaches capacitance values almost $32 \%$ more than the initial one. As previously reported, the introduction of wrinkles into dielectric layer can increases the electrode area and thus the capacitance. ${ }^{40-42}$ Therefore, we believe that wrinkles can be generated in the dielectric film after returning to the flat position, increasing the contact area and subsequently capacitance increases rather than returning to its original values.

Finally, to get a good overview of the new technology developed, comparison with previously reported capacitive sensors was made and the results are depicted in Table 1. Although the pressure sensitivities observed for the new printed self-healing capacitors are not comparable to capacitive sensors prepared from a micropatterned dielectric layer, they do not suffer from potential baseline instability due to air trapping in dielectric layer, large errors due to external noises, and irreproducible sensitivity. ${ }^{43}$ More importantly, the pressure sensitivities observed for the new self-healing capacitive sensors are in the same range as previously reported values for non-healable and/or non-printed capacitors on soft substrates. Additionally, the method employed in this work for the fabrication of the pressure sensors (slot-die coating) is simple and inexpensive, which contrasts with the fabrication of capacitive pressure sensors with high sensitivities that normally require multistep costly fabrication processes.

\section{Conclusion}

In summary, the fabrication of thin film capacitive sensors on a flexible substrate was realized by incorporating self-healing dielectric materials based on metal-ligand supramolecular interaction. The self-healing properties of polymer 1 revealed that the polymer has exceptional autonomous healing rate at room temperature $\left(1.34 \times 10^{5} \mu \mathrm{m}^{3}\right.$ per hour $)$ and reaches values as high as $2.87 \times 10^{5} \mu \mathrm{m}^{3}$ per hour at human body temperature, making it an excellent choice of material for wearable electronic applications. To confirm the potential of polymer $\mathbf{1}$ in self-healing and soft electronics, a series of thin film capacitive sensors were fabricated using slot-die coating on poly(ethyleneterephthalate) (PET) substrate coated with silver ink and PEDOT:PSS top electrode. At first, the bending tolerance of capacitive sensors were examined, and no significant capacitance changes were observed upon bending of devices irrespective of the area of the capacitor. As a capacitive pressure sensor, the pressure sensitivity of the devices was $0.09-2.9$ $\mathrm{kPa}^{-1}$ and $0.02-0.05 \mathrm{kPa}^{-1}$ for $0-1 \mathrm{kPa}$ and $1-2 \mathrm{kPa}$ respectively. In the case of any mechanical damage to the device such as cracking, the device performance proved to be fully restored over 4 hours. However, the concave bending of the devices resulted in a permanent damage to the device and thus reducing the capacitance values by $23-44 \%$. We ascribe this to the low bending tolerance of the silver electrode and its brittle nature.

Considering the rise of the IoT, the inexpensive fabrication of thin film sensing materials and devices at large scale will enable progress in various areas, including electronics and healthcare. The new autonomous self-healing capacitive devices designed in this work not only combine low-cost and rapid fabrication through slot-die coating, but also give access to soft electronics that can be conformable and robust. Given the importance of capacitive sensing, this new sensor design and preparation offers new avenues and opportunities for the expansion of self-healing electronics and their integration to many different platforms.

\section{Funding sources}

This work was supported by NSERC through a Discovery Grants (RGPIN-2017-06611) and the NSERC Green Electronics Network (GreEN) (NETGP 508526-17). S. R.-G. also acknowledges the Canada Foundation for Innovation (CFI), the Ontario Research Fund, and the University of Windsor for financial support.

\section{Author contributions}

All authors contributed to the manuscript. All authors have given approval to the final version of the manuscript.

\section{Conflicts of interest}

There are no conflicts to declare.

\section{Acknowledgements}

The authors thank Dr Chloé Bois and Dr Mariia Zhuldybina of the Institut des communications graphiques et de l'imprimabilité (ICI Montréal) for designing and preparing the PET platform used to fabricate the new sensors. 


\section{References}

1 P. Asghari, A. M. Rahmani and H. H. S. Javadi, Internet of Things applications: A systematic review, Comput. Networks, 2019, 148, 241-261.

2 J. Gubbi, R. Buyya, S. Marusic and M. Palaniswami, Internet of Things (IoT): A vision, architectural elements, and future directions, Future Gener. Comput. Syst., 2013, 29, 1645-1660.

3 M. Swan and Sensor Mania, The Internet of Things, Wearable Computing, Objective Metrics, and the Quantified Self 2.0, J. Sens. Actuator Netw., 2012, 1, 217-253.

4 P. A. Laplante and N. Laplante, The Internet of Things in Healthcare: Potential Applications and Challenges, IT Prof., 2016, 18, 2-4.

5 S. H. Shah and I. Yaqoob, IEEE Smart Energy Grid Engineering (SEGE), 2016, pp. 381-385.

6 J. Kang, J. B.-H. Tok and Z. Bao, Self-healing soft electronics, Nat. Electron., 2019, 2, 144-150.

7 T. F. O'Connor, K. M. Rajan, A. D. Printz and D. J. Lipomi, Toward organic electronics with properties inspired by biological tissue, J. Mater. Chem. B, 2015, 3, 4947-4952.

8 T. Someya, Z. Bao and G. G. Malliaras, The rise of plastic bioelectronics, Nature, 2016, 540, 379-385.

9 M. Berggren and A. Richter-Dahlfors, Organic Bioelectronics, Adv. Mater., 2007, 19, 3201-3213.

10 P. B. J. Onge, M. U. Ocheje, M. Selivanova and S. RondeauGagné, Recent Advances in Mechanically Robust and Stretchable Bulk Heterojunction Polymer Solar Cells, Chem. Rec., 2019, 19, 1008-1027.

11 C. M. Boutry, A. Nguyen, Q. O. Lawal, A. Chortos, S. Rondeau-Gagné and Z. Bao, A Sensitive and Biodegradable Pressure Sensor Array for Cardiovascular Monitoring, Adv. Mater., 2015, 27, 6954-6961.

12 J. Pignanelli, K. Schlingman, T. B. Carmichael, S. RondeauGagné and M. J. Ahamed, A comparative analysis of capacitive-based flexible PDMS pressure sensors, Sens. Actuators, A, 2019, 285, 427-436.

13 X. Wang, T. Li, J. Adams and J. Yang, Transparent, stretchable, carbon- nanotube -inlaid conductors enabled by standard replication technology for capacitive pressure, strain and touch sensors, J. Mater. Chem. A, 2013, 1, 3580-3586.

14 S. Bauer, S. Bauer-Gogonea, I. Graz, M. Kaltenbrunner, C. Keplinger and R. Schwödiauer, 25th Anniversary Article: A Soft Future: From Robots and Sensor Skin to Energy Harvesters, Adv. Mater., 2014, 26, 149-162.

15 S. P. Lacour and D. P. J. Cotton, 16th International SolidState Sensors, Actuators and Microsystems Conference, 2011, pp. 2770-2773.

16 H. Jin, S. Jung, J. Kim, S. Heo, J. Lim, W. Park, H. Y. Chu, F. Bien and K. Park, Stretchable Dual-Capacitor MultiSensor for Touch-Curvature-Pressure-Strain Sensing, Sci. Rep., 2017, 7, 10854.

17 M. N. Tahir, M. U. Ocheje, K. Wojtkiewicz and S. RondeauGagné, Self-Healing Materials: Design and Applications, De Gruyter, 2020.
18 Y. J. Tan, J. Wu, H. Li and B. C. K. Tee, Self-Healing Electronic Materials for a Smart and Sustainable Future, ACS Appl. Mater. Interfaces, 2018, 10, 15331-15345.

19 C. E. Diesendruck, N. R. Sottos, J. S. Moore and S. R. White, Biomimetic Self-Healing, Angew. Chem., Int. Ed., 2015, 54, 10428-10447.

20 F. Herbst, D. Döhler, P. Michael and W. H. Binder, SelfHealing Polymers via Supramolecular Forces, Macromol. Rapid Commun., 2013, 34, 203-220.

21 C.-H. Li, C. Wang, C. Keplinger, J.-L. Zuo, L. Jin, Y. Sun, P. Zheng, Y. Cao, F. Lissel, C. Linder, X.-Z. You and Z. Bao, A highly stretchable autonomous self-healing elastomer, Nat. Chem., 2016, 8, 618-624.

22 Y. Chen, A. M. Kushner, G. A. Williams and Z. Guan, Multiphase design of autonomic self-healing thermoplastic elastomers, Nat. Chem., 2012, 4, 467-472.

23 D. Mozhdehi, S. Ayala, O. R. Cromwell and Z. Guan, SelfHealing Multiphase Polymers via Dynamic Metal-Ligand Interactions, J. Am. Chem. Soc., 2014, 136, 16128-16131.

24 Y. Huang, X. Fan, S.-C. Chen and N. Zhao, Emerging Technologies of Flexible Pressure Sensors: Materials, Modeling, Devices, and Manufacturing, Adv. Funct. Mater., 2019, 29, 1808509.

25 D. Chen and Q. Pei, Electronic Muscles and Skins: A Review of Soft Sensors and Actuators, Chem. Rev., 2017, 117, 11239-11268.

26 Y. Jiang, Z. Liu, Z. Yin and Q. Zheng, Sandwich structured dielectrics for air-stable and flexible low-voltage organic transistors in ultrasensitive pressure sensing, Mater. Chem. Front., 2020, 4, 1459-1470.

27 Z. Yin, M.-J. Yin, Z. Liu, Y. Zhang, A. P. Zhang and Q. Zheng, Solution-Processed Bilayer Dielectrics for Flexible LowVoltage Organic Field-Effect Transistors in PressureSensing Applications, Adv. Sci., 2018, 5, 1701041.

28 Q. Zhang, S. Niu, L. Wang, J. Lopez, S. Chen, Y. Cai, R. Du, Y. Liu, J.-C. Lai, L. Liu, C.-H. Li, X. Yan, C. Liu, J. B.-H. Tok, X. Jia and Z. Bao, An Elastic Autonomous Self-Healing Capacitive Sensor Based on a Dynamic Dual Crosslinked Chemical System, Adv. Mater., 2018, 30, 1801435.

29 T. Wang, Y. Zhang, Q. Liu, W. Cheng, X. Wang, L. Pan, B. Xu and H. Xu, A Self-Healable, Highly Stretchable, and Solution Processable Conductive Polymer Composite for Ultrasensitive Strain and Pressure Sensing, Adv. Funct. Mater., 2018, 28, 1705551.

30 Y. Zhu, L. Lin, Y. Chen, Y. Song, W. Lu and Y. Guo, A selfhealing, robust adhesion, multiple stimuli-response hydrogel for flexible sensors, Soft Matter, 2020, 16, 2238-2248.

31 J. Pignanelli, B. Billet, M. Straeten, M. Prado, K. Schlingman, M. Jalal Ahamed and S. Rondeau-Gagné, Imine and metal-ligand dynamic bonds in soft polymers for autonomous self-healing capacitive-based pressure sensors, Soft Matter, 2019, 15, 7654-7662.

32 J. Pignanelli, Z. Qian, X. Gu, M. J. Ahamed and S. RondeauGagné, Modulating the thermomechanical properties and self-healing efficiency of siloxane-based soft polymers through metal-ligand coordination, New J. Chem., 2020, 44, 8977-8985. 
33 H. Wang, S. Vu, J. Pignanelli, T. A. Fatah, J. F. Trant, S. Mahshid, S. Rondeau-Gagné and M. J. Ahamed, Fabrication and Characterization of Autonomously Self-Healable and Stretchable Soft Microfluidics, Adv. Sustainable Syst., 2021, 2100074.

34 Y. M. Boiko, On the molecular mechanism of self-healing of glassy polymers, Colloid Polym. Sci., 2016, 294, 1237-1242.

35 Y. Yang, X. Ding and M. W. Urban, Chemical and physical aspects of self-healing materials, Prog. Polym. Sci., 2015, 49, 34-59.

36 Y. Li, S. Zhang, N. Hamad, K. Kim, L. Liu, M. Lerond and F. Cicoira, Tailoring the Self-Healing Properties of Conducting Polymer Films, Macromol. Biosci., 2020, 20, 2000146.

37 Y. Li, X. Li, S. Zhang, L. Liu, N. Hamad, S. R. Bobbara, D. Pasini and F. Cicoira, Autonomic Self-Healing of PEDOT:PSS Achieved Via Polyethylene Glycol Addition, Adv. Funct. Mater., 2020, 30, 2002853.

38 S. M. Khan, R. B. Mishra, N. Qaiser, A. M. Hussain and M. M. Hussain, Diaphragm shape effect on the performance of foil-based capacitive pressure sensors, AIP Adv., 2020, 10, 015009.

39 T. Wang, X. Mu, A. B. Randles, Y. Gu and C. Lee, Diaphragm shape effect on the sensitivity of surface acoustic wave based pressure sensor for harsh environment, Appl. Phys. Lett., 2015, 107, 123501.

40 S. Baek, H. Jang, S. Y. Kim, H. Jeong, S. Han, Y. Jang, D. H. Kim and H. S. Lee, Flexible piezocapacitive sensors based on wrinkled microstructures: toward low-cost fabrication of pressure sensors over large areas, RSC Adv., 2017, 7, 39420-39426.

41 J. Cui, B. Zhang, J. Duan, H. Guo and J. Tang, Flexible Pressure Sensor with Ag Wrinkled Electrodes Based on PDMS Substrate, Sensors, 2016, 16, 2131.

42 G. Yu, J. Hu, J. Tan, Y. Gao, Y. Lu and F. Xuan, A wearable pressure sensor based on ultra-violet/ozone microstructured carbon nanotube/polydimethylsiloxane arrays for electronic skins, Nanotechnology, 2018, 29, 115502.

43 J. Qin, L.-J. Yin, Y.-N. Hao, S.-L. Zhong, D.-L. Zhang, K. Bi, Y.-X. Zhang, Y. Zhao and Z.-M. Dang, Flexible and Stretchable Capacitive Sensors with Different Microstructures, Adv. Mater., 2021, 2008267.

44 D. Maddipatla, X. Zhang, A. K. Bose, S. Masihi, B. B. Narakathu, B. J. Bazuin, J. D. Williams, M. F. Mitchell and M. Z. Atashbar, A Polyimide Based Force Sensor Fabricated Using Additive ScreenPrinting Process for Flexible Electronics, IEEE Access, 2020, 8, 207813.

45 M. Schouten, R. Sanders and G. Krijnen, IEEE Sensors, 2017, pp. 1-3.

46 S. Wan, H. Bi, Y. Zhou, X. Xie, S. Su, K. Yin and L. Sun, Graphene oxide as high-performance dielectric materials for capacitive pressure sensors, Carbon, 2017, 114, 209-216.

47 B. C.-K. Tee, C. Wang, R. Allen and Z. Bao, An electrically and mechanically self-healing composite with pressure- and flexion-sensitive properties for electronic skin applications, Nat. Nanotechnol., 2012, 7, 825-832. 